[Frontiers In Bioscience, Landmark, 23, 27-35, January 1, 2018]

\title{
Leader sequences of coronavirus are altered during infection
}

\author{
Li Liu', Wei Chen ${ }^{2}$
}

${ }^{1}$ Department of Microbiology, Institute of Basic Medical Sciences, Chinese Academy of Medical Sciences and Peking Union Medical College, Beijing 100005, China, ${ }^{2}$ Beijing Institute of Biotechnology, No. 20 Dongdajie Street, Fengtai District, Beijing 100071, China

\section{TABLE OF CONTENTS}

\section{Abstract}

2. Introduction

3. Materials and methods

3.1. Cell culture, SARS-CoV strain and viral infection

3.2. The leader sequence information of SARS-CoV and MHV

3.3. Primer designs and sequence information

3.4. RNA isolation and RT-PCR analysis

\section{Results}

4.1. Identification of a novel sgmRNA from HKU-39849 strain of SARS-CoV

4.2. Analysis of the leader and TRS sequences of sgmRNAs derived from both SARS-CoV and MHV

4.3. The different patterns of leader alterations in MHV versus SARS-CoV genome

5. Discussion

6. Acknowledgments

7. References

\section{ABSTRACT}

The life cycle of Severe Acute Respiratory Syndrome Coronavirus (SARS-CoV) involves a unique process called discontinuous transcription by which a set of $3^{\prime}$ coterminal subgenomic mRNAs (sgmRNA) with identical 5 ' leader sequences can be generated. The current study demonstrates that the replication intermediates of minus strand of subgenomic RNA (sgRNA) can be readily recovered from SARS-CoV infected cells. A novel sgmRNA (M-1) was identified as a short version of membrane (M) gene. Transcriptional regulatory sequences (TRS) of SARS-CoV and Mouse Hepatitis Virus (MHV) sgmRNAs contain a species specific core element (CE). The sizes of leader sequences in MHVs vary not only in different viral strains but also among different genes in the same strain. Leader alterations such as deletion and nucleotide substitution were observed in MHVs, while a dynamic one-orientation "sequential deletion" was found among the leaders of 76 SARS-CoV isolates. These results imply that the leader sequence of coronavirus might be unstable and leader alterations during SARS-CoV transmission in humans might have negative impact on its viral infectivity.

\section{INTRODUCTION}

Severe Acute Respiratory Syndrome (SARS) coronavirus is the causing pathogen responsible for the outbreak of the fatal atypical pneumonia worldwide in 2002-2003 (1-3). Phylogenetic analysis initially showed that SARS-CoV did not belong to any group of known coronaviruses, but could be classified as group $2 b$ virus based on the conservation of the carboxyl terminal domain of spike protein (4). Due to the recent expansion of novel coronaviruses, the viruses have been classified into 4 genera (alphacoronavirus, betacoronavirus, detacoronavirus and gammacoronavirus) based on phylogenetic analysis and the conserved sequences of the non structural proteins, while SARS-CoV was re-grouped as betacoronavirus (5).

SARS-CoV is a single-stranded positive RNA virus with a genome size around 29,700 nucleotides (nt), and presents a typical coronavirus genomic organization $(6,7)$ that encodes for at least 14 open reading frames (ORFs). The first two thirds of SARSCoV genome produces two large overlapped ORFs (ORF 1a and ORF 1b). The other 12 ORFs are generated from the rest one third of viral genome. After infecting the cells via membrane fusion and endocytosis, SARS-CoV releases its genetic content into the cytoplasm and immediately synthesizes the two large polyproteins pp1a and pp1ab from ORF 1a and ORF $1 \mathrm{~b}$ involving a process called programmed 
-1 ribosomal frame-shift (8). The polyproteins pp1a and pp1ab are subsequently subjected to proteolytic processing for the generation of 16 nonstructural proteins that some of them are essential for viral genome replication and viral RNA synthesis (9). The ORFs from 5' to 3 ' direction located at the 3 ' $1 / 3$ of SARS-CoV genome encode four major structural genes S (ORF 2), E (ORF 4), M (ORF 5) and N (ORF 9). The interspaced are the rest of ORFs that encode the other 8 accessory proteins which are marked as group specific gene products since they share almost no sequence homology with other groups of coronaviruses (10). Two ORFs, ORF3a and ORF3b, are located between $S$ and $E$ genes, ORF $6,7 \mathrm{a}, 7 \mathrm{~b}$, $8 a$ and $8 b$ are between $M$ and $N$ genes (10-12), while $\mathrm{N}$ gene also contains an internal ORF $9 \mathrm{~b}$ protein to shuttle between cytoplasm and nucleus $(13,14)$ and to induce anti innate immune response (15).

Upon the production of viral replicase proteins, SARS-CoV undergoes the second stage of viral infection by initiating viral genomic transcription and replication for the generation of the positive genome sized RNA as well as a nested set of 3' coterrminal subgenomic mRNAs (sgmRNA) with an identical 5' leader sequence via a unique mechanism called discontinuous transcription $(16,17)$. A double membrane vesicle (DMV) formed in SARS-CoV infected cells is believed to be the site of viral replication and transcription (18). A replication and transcription complex (RTC) containing viral replicase-transcriptase proteins plus other viral proteins as well as cellular proteins is associated with DMV in which both genome size and subgenomic RNA are synthesized $(16,19)$. For synthesizing the minus stranded subgenomic RNA, RTC processes along the positive RNA genome template from its 3' end, and then either reads the body TRS (TRS-B) upstream of each ORF as an attenuation signal and relocate the nascent RNA to copy the genomic leader sequence by recognizing the 3' leader TRS (TRS-L), or continuously transcribes through to meet next TRS-B signal. The production of minus stranded RNA will serve as replication templates for the subsequent synthesis of mRNA.

Nucleotide substitution, insertions and sequence deletions were frequently observed in different isolates of SARS-CoV (20-25). We speculate that all these changes may result from the adaption of SARS-CoV in human during disease transmission. Some sequence alternations have been shown to occur increasingly in SARS patients (19) and may affect viral infectivity (24). In addition, the strain variations in mouse hepatitis viruses (MHVs), a betacoronavirus, may also influence viral infectivity and pathogenesis (26). For example, a serine-to-glycine change at position 310 of the S protein may account for the more neurovirulent phenotype in MHV-JHM strain (27). A strain variant isolated from persistently infected mouse model obtained increased virulence and infectivity. Subsequent sequencing analysis on this viral strain uncovered a 29 amino acid deletion at S protein that may account for the loss of its cytotoxic $T$ cell epitope, indicating that MHV virus adaptive mutation may be important for viral immune escape (28).

In this study, the leader sequences of both SARS-CoV and MHV were comparatively analyzed. The different patterns of the strain-specific leader variations among the isolates of both SARS-CoV and MHV were apparent. Sequence alignment on the genomic leader sequences derived from 76 isolates of complete SARS-CoV genomes demonstrated a "sequential deletion" process, while the alterations of the leader sequences derived from MHV isolates most often presented with single nucleotide substitution and short sequence deletions. Thus, the current study indicates that the leader sequences of coronaviruses might be unstable during epidemic and disease transmission.

\section{MATERIALS AND METHODS}

\subsection{Cell culture, SARS-CoV strain and viral infection}

Vero E6 cells were cultured in Dulbecco's modified Eagle's medium (DMEM) supplemented with $10 \%$ heat-inactivated fetal bovine serum supplemented with $5 \% \mathrm{CO}_{2}$ at $37^{\circ} \mathrm{C}$. SARS-CoV strain HKU-39849 (29) (provided by Dr. KY Yuen, The University of Hong Kong) was propagated in Vero E6 cells in P3 laboratory. The virus was released from the infected cells by three cycles of freezing and thawing. $2 \times 10^{6}$ cells were plated onto $60 \mathrm{~mm}$ dish and infected with $1 \times 10^{6} \mathrm{MOI}$ of SARS-CoV (HKU-39849) in DMEM medium without FBS for 2 hours at $37^{\circ} \mathrm{C}$. Cells were washed once with phosphate buffered saline (1xPBS, $\mathrm{pH} 7.4$.), added $4 \mathrm{ml}$ of complete medium, and incubated for another 24 hours at $37^{\circ} \mathrm{C}$ before harvesting. All operations were performed in a bio-safety P3 laboratory.

\subsection{The leader sequence information of SARS-CoV and MHV}

The genomic leader sequences of MHV JHM, MHV JHM.IA, MHV-A59, MHV S, MHV-1, MHV-2, and MHV-3 were deduced from their complete genomic cDNA sequences with GenBank accession numbers NC_006852, FJ647226, NC_001846, GU593319, FJ647223, AF201929 and FJ647224, respectively. The GenBank accession numbers for the leader containing sgmRNAs $\mathrm{S}, \mathrm{M}$, and $\mathrm{N}$ of MHV JHM were X04797, X04223.1, and X00990.1, respectively, and for the leader containing sgmRNAs $S, M$ and $N$ of MHV A59 were M18379.1, M25906 and M25907.1, respectively. The deduced genomic leader sequences from 76 strains of SARS-CoV were isolated from 
GenBank database according to the complete genomic sequence information of the selected viral strains with the GenBank accession numbers listed below: GZ02, AY390556; HZS2-Bb, AY395004; ZS-C, AY395003; CUHK-LC5, AY395002; CUHK-LC4, AY395001; CUHK-LC3, AY395000; CUHK-LC2, AY394999; ZSA, AY394997; ZS-B, AY394996; HSZ-Cc, AY394995; HSZ-Bc, AY394994; HGZ8L2, AY394993; HZS2-C, AY394992; HZS2-Fc, AY394991; HZS2-E, AY394990; HZS2-D, AY394989; JMD, AY394988; HZS2-Fb, AY394987; HSZ-Cb, AY394986; TW3, AY502926; BJ04, AY279354; HGZ8L1-A, AY394981; HGZ8L1-B, AY394982; ZS-C, AY395003; HSZ2-A, AY394983; GZC, AY394979; Tor2, NC_004718; BJ01, AY539954; WHU, AY394850; NS-1, AY508724; TW10, AY502923; TW2, AY502925; ShanghaiQXC1, AY463059; ZJ01, AY286320; ShanghaiQXC2, AY463060; GD69, AY313906; FRA, AY310120; SoD, AY461660; Sino111, AY485277; CUHK-AG03, AY345988; CUHKAG02, AY345987; CUHK-AG01, AY345986; CUHKSu10, AY282752; PUMC03, AY357076; PUMC02, AY357075; PUMC01, AY350750; GZ50, AY304495; SZ16, AY304488; SZ3, AY304486; AS, AY427439; HSR 1, AY323977; Sin2774, AY283798; HKU-39849, AY278491; GD01, AY278489; TWC2, AY362698; Sin2748, AY283797; Sin2679, AY283796; Urbani, AY278741; ZMY 1, AY351680; TWY, AP006561; TWS, AP006560; CUHK-W1, AY278554; TC3, AY348314; TC2, AY338175; TC1, AY338174; TWC, AY321118; Frankfurt 1, AY291315; Sino3-11, AY485278; BJ03, AY278490; BJ02, AY278487; ZJ01, AY297028; TW1, AY291451.

\subsection{Primer designs and sequence information}

The most 5' end of the complete genome of SARS-CoV strain HKU-39849 was served as the template for the design of the common forward primer, while reversed primers were deduced from the 3' genomic sequences of the structural genes (S, M, E, and N). The common forward primer was 5'-atattaggttttacctacc-3'. The reversed primers for the structural protein genes were 5'-ccatgcatagacagaagggaa-3' (S), 5'-ttactgtactagcaaagcaa-3' (M), 5'-ttagaccagaagatcagga-3' (E), and 5'-gctattaaaatcacatgggga-3' (N). The amplifications of different structural genes of SARS-CoV were conducted by RT-PCR. The reaction products were subcloned into pGEM-T-easy Vector and subsequently sequenced.

\subsection{RNA isolation and RT-PCR analysis}

Total RNAs from $5 \times 10^{6}$ SARS-CoV infected cells were extracted with $1 \mathrm{ml}$ Trizol (Invitrogen) plus $200 \mu \mathrm{l}$ chloroform. The supernatant was precipitated with an equal volume of isoproponal. Then, RNA pellet was resuspended in sterile water. About 1-2 mg RNAs was used as templates for one-step RTPCR analysis (Takara Biotechnology) as described in the manual. The reaction mixture was first incubated at $50^{\circ} \mathrm{C}$ for 30 minutes for reverse transcription, and then denatured at $94^{\circ} \mathrm{C}$ for 2 minutes before PCR cycles. Thirty cycles of PCR was conducted at following condition: $94^{\circ} \mathrm{C}$ for 30 seconds, $55^{\circ} \mathrm{C}$ for 30 seconds, and $72^{\circ} \mathrm{C}$ for 2 minutes. The reaction products were extended at $72^{\circ} \mathrm{C}$ for 10 minutes before storing at $4^{\circ} \mathrm{C}$.

\section{RESULTS}

\subsection{Identification of one novel sgRNA from} HKU-39849 strain of SARS-CoV

The life cycle of coronavirus involves a unique discontinuous transcription with the production of the replication intermediates, the subgenomic minus strand RNAs. To identify these replication intermediates, specific 5 ' primers were designed to amplify the sgRNAs of SARS-CoV HKU-39849 strain for S, N, M and E genes, respectively (Figure $1 \mathrm{~A}$ and 1B). RT-PCR and sequencing analysis showed that the replication intermediates of minus strand RNAs were indeed produced for $\mathrm{S}, \mathrm{N}, \mathrm{M}, 3 \mathrm{a} / 3 \mathrm{~b}$ and $\mathrm{E}$ genes (Figure 2). Unexpectedly, one novel sgRNA (named $\mathrm{M}-1$ ) with minus strand of replication intermediate could also be detected (Figure 1A and 1B). Sequencing analysis reveals that $M-1$ mRNA is a short version of $M$ sgmRNA that only contains the last $145 \mathrm{nt}$ of M coding sequence (Figure 1C).

4.2. Analysis of the leader and TRS sequences of sgmRNAs derived from both SARS-CoV and MHV

The leader-body fusion sites of SARSCoV and MHV were uncovered by sequence comparison between the 5' end of sgmRNA and its complete genomic RNA. The fusion sites for $S$, $\mathrm{N}, \mathrm{M}, \mathrm{M}-1,3 \mathrm{a} / 3 \mathrm{~b}$ and $\mathrm{E}$ genes of SARS-CoV were CUAAACGAAC, UAAACGAAC, UCUAAACGAAC, ACGAAC, UAAACGAACUU and ACGAACUU (underline indicates the core element (CE)), respectively (Figure 2 ). The fusion sizes varied from 6 to 11 nucleotides (nt) which resulted in the variants of leaders from 72 to 74 nt (Figure 2). For MHVs, the size of leader sequence varies not only in different strains but also among different genes in the same strain (Figure 3). The TRS sequences of MHVs were conserved for the same genes in different strains, while the sizes of TRS apparently varied (Figure 3). For example, the TRS for $S$ gene is composed of 10 nt with a single pentanucleotides (UCUAA) core element, while the TRS sequences for both $\mathrm{M}$ and $\mathrm{N}$ genes contained a tandem repeat of CE (Figure 3 ). Moreover, the sizes of leader sequences varied from $76 \mathrm{nt}$ to $80 \mathrm{nt}$ (Figure $5 \mathrm{~A}$ ) and from $71 \mathrm{nt}$ to $80 \mathrm{nt}$ for MHV JHM and MHV A59 (Figure 3), respectively. 

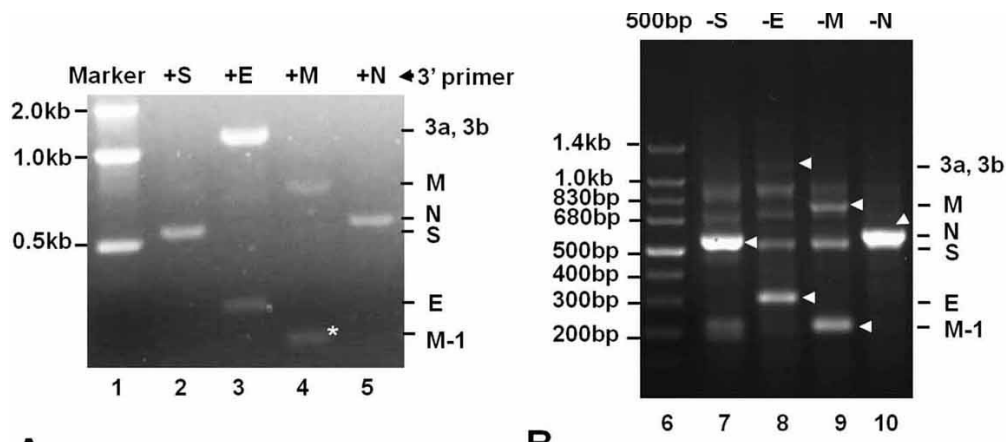

A

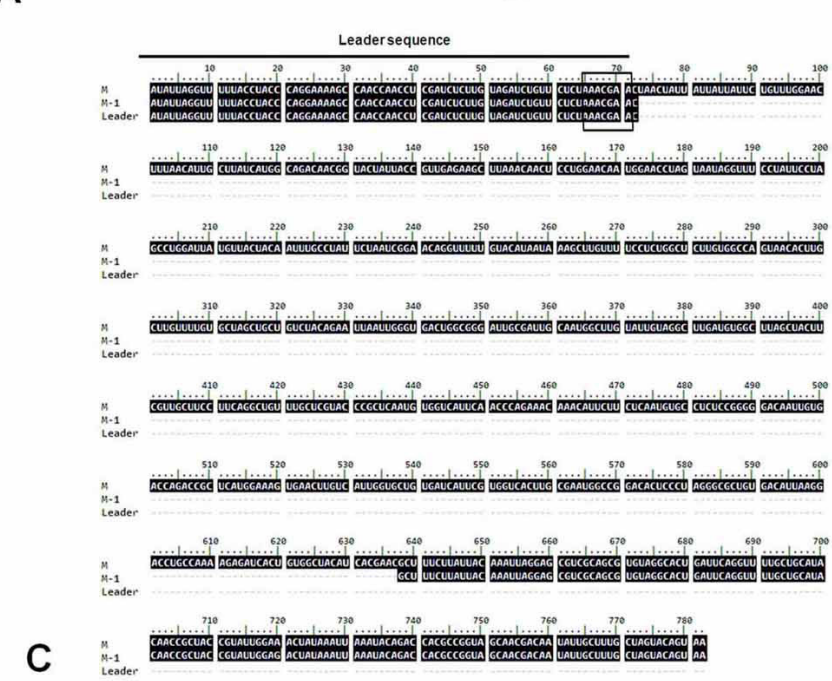

Figure 1. Evidence for the presence of both positive and negative subgenomic RNAs during SARS-CoV infection. Total RNAs were isolated from SARS$\mathrm{CoV}$ infected Vero E6 cells after $48 \mathrm{hr}$ post-infection. For the detection of either positive (A) or negative sgRNAs (B), the first stranded cDNAs were synthesized independently by using oligo $\mathrm{dT}$ or anti leader $\left(P_{L}\right)$ as primer, respectively, and subsequently PCR reactions were amplified using $S, M, N$ and E specific primers. The suspected bands (white arrows) were isolated from agarose gel and subjected to sequencing analysis. (C) Sequence alignment on M sgmRNA, M-1 sgmRNA and leader sequence by ClutalW. Box indicated the sgmRNA conserved TRS core element.

\section{$\underline{\text { CS }} \quad \underline{\text { TRS size }} \quad \underline{\text { Leader size }}$}

\begin{tabular}{|c|c|c|c|}
\hline S-5'genome & GUAGGCUUAUCAUUAGAGAAAACAACAGAGUUGUGGUUUCAAGUGAUAUUCUUGUUAACAACUAAACGAACAUG & & \\
\hline HKU leader & AUAUUAGGUUUUUACCUACCCAGGAAAAGCCAACCAACCUCGAUCUCUUGUAGAUCUGUUCY UCUA & 10nt & $72 \mathrm{nt}$ \\
\hline S sgmRNA & AUAUUAGGUUUUUACCUACCCAGGAAAAGCCAACCAACCUCGAUCUCUUGUAGAUCUGUUCUCUAA & & \\
\hline N-5' genome & GUGUUGGUACCUUCAUGAAGGUCACCAAACUGCUGCAUUUAGAGACGUACUUGUUGUUUAAAUA,ACGAACAAAUUAAAAUG & & \\
\hline HKU leader & AUAUUAGGUUUUUACCUACCCAGGAAAAGCCAACCAACCUCGAUCUCUUGUAGAUCUGUUCUOC & 9nt & $72 \mathrm{nt}$ \\
\hline $\mathrm{N}$ sgmRNA & AUAUUAGGUUUUUACCUACCCAGGAAAAGCCAACCAACCUCGAUCUCUUGUAGAUCUGUUCUCUAACGAACAAAUUAAAACG & & \\
\hline M-5'genome & GUUUACGUCUACUCGCGUGUUAAAAAUCUGAACUCUUCUGAAGGAGUUCCUGAUCUUCUGGUCUA $\sqrt{A C G A A Q U A A C U-39 n-A L G}$ & & \\
\hline HKU leader & AUAUUAGGUUUUUACCUACCCAGGAAAAGCCAACCAACCUCGAUCUCUUGUAGAUCUGUUCUCUAA & $12 \mathrm{nt}$ & $73 \mathrm{nt}$ \\
\hline M sgmRNA & AUAUUAGGUUUUUACCUACCCAGGAAAAGCCAACCAACCUCGAUCUCUUGUAGAUCUGUUCUCUAAACGAACUAACU-39nt-AUG & & \\
\hline $\begin{array}{l}\text { M-1 genome } \\
\text { HKU leader }\end{array}$ & $\begin{array}{l}\text { ATGGCCGGACACTCCCTAGGGCGCTGTGACATTAAGGACCTGCCAAAAGAGATCACTGTGGCTACATCACGAACGCUU-15int-ALG } \\
\text { AUAUUAGGUUUUUACCUACCCAGGAAAAGCCAACCAACCUCGAUCUCUUGUAGAUCUGUUCUCUAA }\end{array}$ & 6 nt & $72 \mathrm{nt}$ \\
\hline M-1 sgmRNA & AUAUUAGGUUUUUACCUACCCAGGAAAAGCCAACCAACCUCGAUCUCUUGUAGAUCUGUUCUCUAAACGAACGCUU-15int-ALG & & \\
\hline $3 \mathrm{a} / 3 \mathrm{~b}$ genome & UGCUGCAAGUUUGAUGAGGAUGACUCUGAGCCAGUUCUCAAGGGUGUCAAAUUACAUUACACAUAADCGAAQUUAUG & & \\
\hline HKU leader & AUAUUAGGUUUUUACCUACCCAGGAAAAGCCAACCAACCUCGAUCUCUUGUAGAUCUGUUCU UAAACGAACUUUAA & 9nt & 74 nt \\
\hline a/3b sgmRNA & AUAUUAGGUUUUUACCUACCCAGGAAAAGCCAACCAACCUCGAUCUCUUGUAGAUCUGUUCUCUAAACGAAGUUALGGAU & & \\
\hline E genome & UGGAUCCCAUUUAUGAUGAGCCGACGACGACUACUAGCGUGCCUUUGUAAGCACAAGAAAGUGAGUACGAACUUAAIG & & \\
\hline HKU leader & ACCCAGGAAAAGCCAACCAACCL & $8 \mathrm{nt}$ & $74 \mathrm{nt}$ \\
\hline
\end{tabular}

Figure 2. The heterogeneity of the leader sequences in different sgmRNAs of SARS-CoV. Each sequenced sgmRNA such as S, N, M, M-1, 3a/3b or E was aligned with both the leader sequence and its respective genome sequence by ClutalW program. Underlines represent the identified TRS elements overlapped among viral genome sequence, 5 ' leader sequence and the sgmRNA. Box indicates the TRS core element (CE). The sizes of both leader sequence and TRS are indicated at the right panel. 


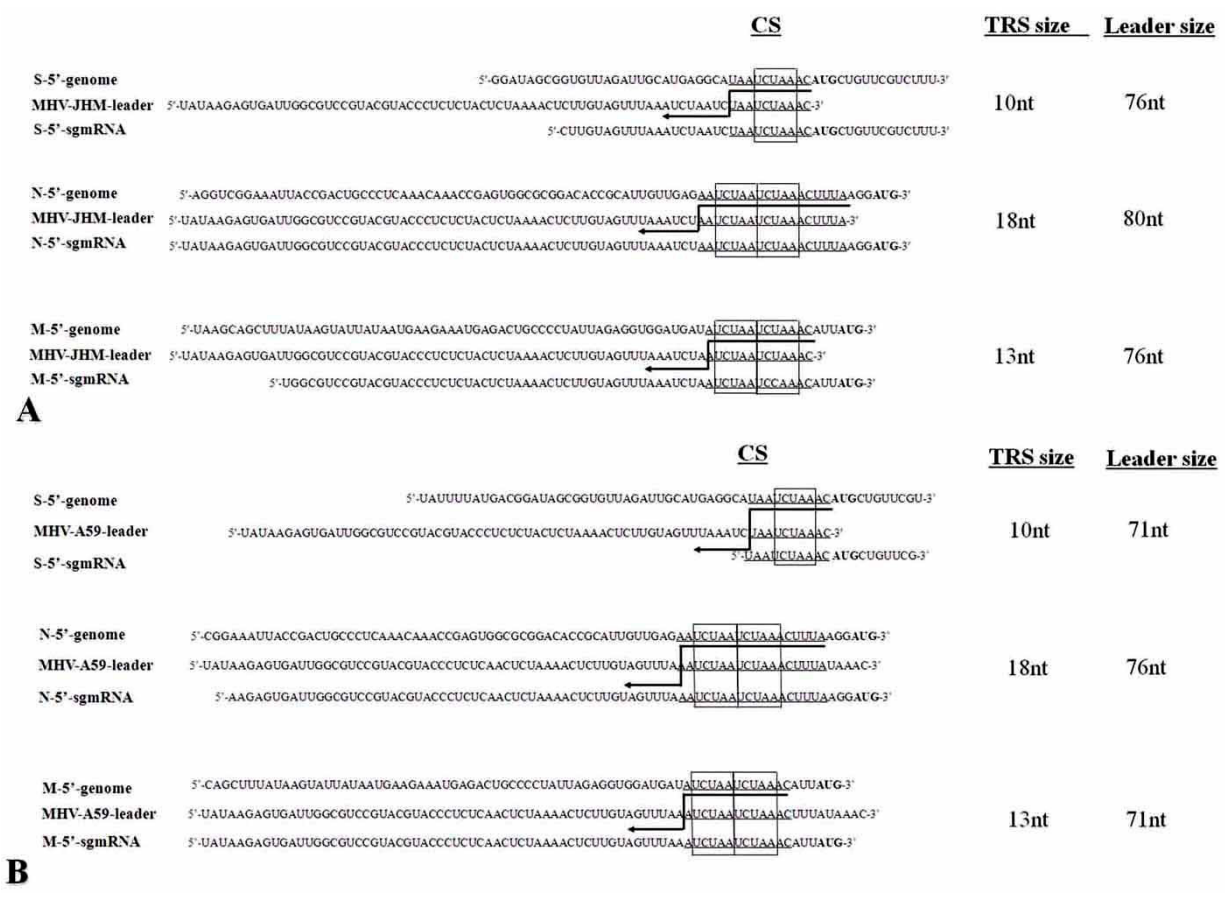

Figure 3. The heterogeneity of the leader sequences in different strains of MHV. ClutalW analysis on MHV JHM and MHV A59 strains is shown in (A) and (B), respectively. Each identified sgmRNA such as S, N and $\mathrm{M}$ was aligned with both the leader sequence and its respective genome sequence. Underlines indicate the overlapped TRS elements among the genome sequence of a specific viral gene, the leader sequence and its corresponding sgmRNA. Box indicates the TRS core element (CE). For each identified sgmRNA, the sizes of leader sequence and TRS were indicated at the right panel.

\subsection{The different patterns of leader alterations in MHV versus SARS-CoV genome}

Although the role of the leader sequence in regulating coronaviral transcription is well known, it is still unclear how sequence conservation at the 5 ' end that coronavirus has during viral transmission. In order to address this issue directly, the leader sequences from seven MHV strains were isolated from GenBank: A59, JHM.IA, JHM, strain 1, strain 2, strain 3 and strain S, with access numbers NC 001846, FJ647226, NC 006852, FJ647223, AF201929, FJ647224 and GU593319, respectively. ClustalW analysis showed that four types of alterations were presented at the $5^{\prime}$ end of MHV genomes including 5' deletion, internal deletion, nucleotide substitution and nucleotide insertion (Figure 4A). The heterogeneous uses of the pentanucleotide CE were observed in the leaders of MHV sgmRNAs (30). Our sequence analysis indicates that this heterogeneity in CE repeats might directly caused by the genomic alterations of MHVs in which some strains such as MHV-2, MHV-A59 and MHV-S only present two rather than three tandem repeats of the pentanucleotide CE (Figure 4A). This type of alteration does not affect the correct fusion site formation but reduces the leader size of the respective sgmRNAs (Figure 3).

The next question that we asked was whether the leader alterations of SARS-CoV were similar to that of MHV. To address this question directly, the leader sequences of 76 isolates of SARS-CoV were collected from GenBank. We only selected those leader sequences that had been marked as complete rather than partially sequenced SARS-CoV genomes from the NCBI database. Intriguingly, ClustalW alignment depicted in Figure 4B shows that SARS-CoVs might undergo "sequential deletions" at their 5' ends of genomes in which some viral strains isolated later stage such as ShanghaiQXC1 and ShanghaiQXC2 (isolated on May 25, 2003, personnel communication with Dr. Zhenghong Yuan) completely lost their genomic leader sequences. In contrast to MHV, only three out of 76 SARS-CoV strains have single nucleotide substitution in their leader regions (Figure 6B). Thus, a lower substitution rates and a higher deletion rates might be associated with SARS-CoV leader sequences during viral passage in the host.

\section{DISCUSSION}

In this study, the leader sequences isolated from the complete genomes of both SARS-CoV and MHV were comparatively studied. The results indicate that leader alterations might be common during coronaviral infection. The extent of sequence identity between TRS-L and TRS-B in SARS-CoV and MHV sgmRNA varied markedly, but all contained a species specific core element (CE). The current data also show that sequence deletions and nucleotide substitutions were frequently 


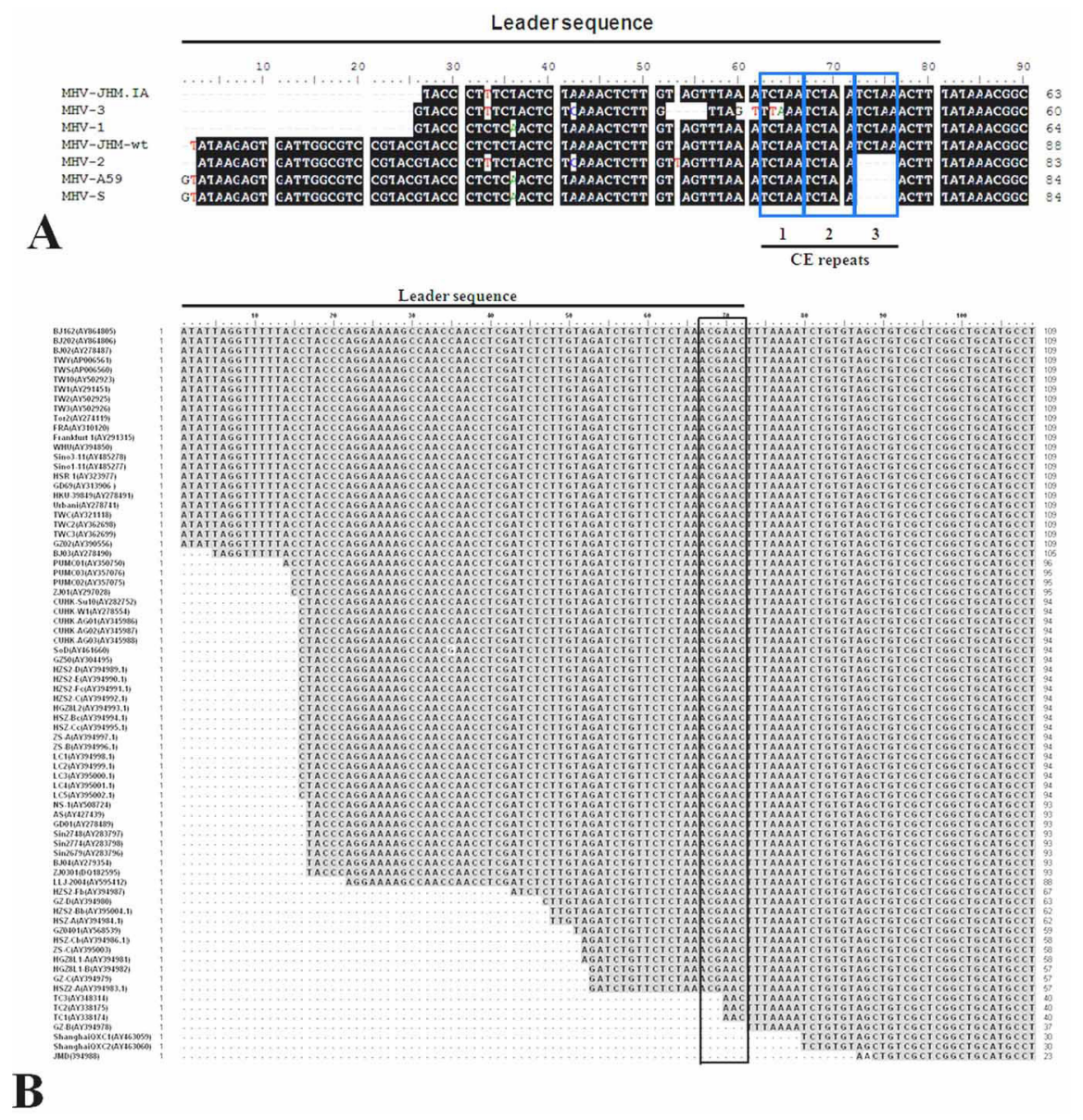

Figure 4. Sequence alignment reveals the patterns of the leader alterations of MHV and SARS-CoV. (A) The 5' ends of genomic sequences were isolated from completely sequenced genomes of seven MHV strains (JHM-IA, MHV1, MHV2, MHV3, MHVS, A59 and JHM) and subjected to sequence alignment with ClutalW. The leader sequence is indicated by a line above the scale. CE repeats were boxed. (B) One-orientation and "sequential deletions" occurred at the 5' ends of genomic leader sequences of SARS-CoV. The genomic leader sequences derived from the 76 isolates of the completely sequenced SARS-CoV genomes were aligned with ClustalW program. The shaded area indicates the identical nucleotides, while the core element is boxed. Dash lines represent the deleted nucleotides. The bold line marks the genomic leader sequence.

observed in MHV genomes, while one orientation deletions at the 5 ' end of leader sequences might be the characteristic feature of SARS-CoV isolates.

Our study uncovered a novel sgmRNA named M-1 from SARS-CoV infected cells. M1 should be a real viral gene product since the minus strand replication intermediate could be readily detected (Figure 1B). Previous reports showed that the extent of sequence identity of TRS-L and TRS-Bs ranged from 7 to 18 nucleotides (31). However, current study revealed that the functional body-fusion junction can be as short as hexanucleotide (ACGAAC) showing in Figure $1 \mathrm{~B}$ for $\mathrm{M}-1$. Among the different species of coronaviruses, MHV might be able to generate the longest sequence identity (18 nt) between TRS-L and TRS-B. A pentanucleotide core element (UCUAA) could be repeated for three times at the 3 ' leader sequence in some strains of MHV such as MHV JHM. Deleting one copy of this core element in the leader sequence of MHV A59 strain reduces the size of the leader sequence but retains the sequence identity of TRS-L and TRS-B duplex. MHV JHM strain is believed to be more virulent than MHV A59 strain $(32,33)$. MHV JHM carrying three copies of CE can cause acute fetal encephalitis and chronic demyelination, whereas MHV A59 with two copies of CE only induces hepatitis, mild encephalitis and subacute demyelination. Since the TRS-L/TRS-B duplex formation could serve as a driving force for viral transcription (31), we speculate that reducing CE copy number may impair viral transcription and replication, and therefore eventually cause the reduced infectivity in some MHV strains.

The "sequential deletions" at the 5' genomic RNA of SARS-CoV may also generate a negative impact on the viral life cycle during viral transmission. In addition, earlier studies showed that the genomic RNAs isolated from patients in late epidemic stage presented larger pieces of genomic deletions $(20,34)$. We speculate that, when SARS-CoV enters the human body, it has to deal with the high pressure delivered by 
the host immune system. This kind of pressure may be loaded onto the viral leader once a time during its replication or transmission, and eventually leads to its complete loss during late stage of epidemic.

\section{ACKNOWLEGEMENTS}

This work was supported by grants from National Natural Science Foundation of China (grant No. 81272230 and 81550030 ).

\section{REFERENCES}

1. T. G. Ksiazek, D. Erdman, C. S. Goldsmith, S. R. Zaki, T. Peret, S. Emery, S. Tong, C. Urbani, J. A. Comer, W. Lim, P. E. Rollin, S. F. Dowell, A. E. Ling, C. D. Humphrey, W. J. Shieh, J. Guarner, C. D. Paddock, P. Rota, B. Fields, J. DeRisi, J. Y. Yang, N. Cox, J. M. Hughes, J. W. LeDuc, W. J. Bellini and L. J. Anderson: A novel coronavirus associated with severe acute respiratory syndrome. $N$ Engl J Med, 348(20), 1953-66 (2003)

DOI:10.1056/NEJMoa030781

PMid:12690092\$\$

2. J. S. Peiris, S. T. Lai, L. L. Poon, Y. Guan, L. Y. Yam, W. Lim, J. Nicholls, W. K. Yee, W. W. Yan, M. T. Cheung, V. C. Cheng, K. H. Chan, D. N. Tsang, R. W. Yung, T. K. Ng and K. Y. Yuen: Coronavirus as a possible cause of severe acute respiratory syndrome. Lancet, 361(9366), 1319-25 (2003)

DOI: 10.1016/S0140-6736(03)13077-2

3. J. S. Peiris, K. Y. Yuen, A. D. Osterhaus and K. Stohr: The severe acute respiratory syndrome. N Engl J Med, 349(25), 2431-41 (2003)

DOI: 10.1056/NEJMra032498

PMid:14681510

4. M. Eickmann, S. Becker, H. D. Klenk, H. W. Doerr, K. Stadler, S. Censini, S. Guidotti, V. Masignani, M. Scarselli, M. Mora, C. Donati, J. H. Han, H. C. Song, S. Abrignani, A. Covacci and R. Rappuoli: Phylogeny of the SARS coronavirus. Science, 302(5650), 1504-5 (2003)

DOI: $10.1126 /$ science.302.5650.1504b

PMid:14645828

5. R. J. de Groot, S. C. Baker, R. S. Baric, D. A. Brian, L. Enjuanes, A. E. Gorbalenya, K. V. Holmes, S. Perlman, L. L. Poon, P. J. M. Rottier, P. J. Talbot, P. C. Y. Woo and J. Ziebuhr: Family Coronaviridae. In: Virus Taxonomy, the 9th Report of the International Committee on Taxonomy of Viruses, , King,
A.M.Q., Adams, M.J., Carstens, E.B. \& E.J Lefkowitz, Eds. (Academic Press), pp. 806828. (2012)

6. M. A. Marra, S. J. Jones, C. R. Astell, R. A. Holt, A. Brooks-Wilson, Y. S. Butterfield, J. Khattra, J. K. Asano, S. A. Barber, S. Y. Chan, A. Cloutier, S. M. Coughlin, D. Freeman, N. Girn, O. L. Griffith, S. R. Leach, M. Mayo, H. McDonald, S. B. Montgomery, P. K. Pandoh, A. S. Petrescu, A. G. Robertson, J. E. Schein, A. Siddiqui, D. E. Smailus, J. M. Stott, G. S. Yang, F. Plummer, A. Andonov, H. Artsob, N. Bastien, K. Bernard, T. F. Booth, D. Bowness, M. Czub, M. Drebot, L. Fernando, R. Flick, M. Garbutt, M. Gray, A. Grolla, S. Jones, H. Feldmann, A. Meyers, A. Kabani, Y. Li, S. Normand, U. Stroher, G. A. Tipples, S. Tyler, R. Vogrig, D. Ward, B. Watson, R. C. Brunham, M. Krajden, M. Petric, D. M. Skowronski, C. Upton and R. L. Roper: The Genome sequence of the SARS-associated coronavirus. Science, 300(5624), 1399-404 (2003)

DOI: $10.1126 /$ science. 1085953

PMid:12730501

7. P. A. Rota, M. S. Oberste, S. S. Monroe, W. A. Nix, R. Campagnoli, J. P. Icenogle, S. Penaranda, B. Bankamp, K. Maher, M. H. Chen, S. Tong, A. Tamin, L. Lowe, M. Frace, J. L. DeRisi, Q. Chen, D. Wang, D. D. Erdman, T. C. Peret, C. Burns, T. G. Ksiazek, P. E. Rollin, A. Sanchez, S. Liffick, B. Holloway, J. Limor, K. McCaustland, M. Olsen-Rasmussen, R. Fouchier, $S$. Gunther, A. D. Osterhaus, C. Drosten, M. A. Pallansch, L. J. Anderson and W. J. Bellini: Characterization of a novel coronavirus associated with severe acute respiratory syndrome. Science, 300(5624), 1394-9 (2003)

DOI: 10.1126/science.1085952

PMid:12730500

8. E. P. Plant and J. D. Dinman: The role of programmed-1 ribosomal frameshifting in coronavirus propagation. Front Biosci, 13, 4873-81 (2008)

DOI: $10.2741 / 3046$

PMid:18508552 PMCid:PMC2435135

9. V. Thiel, K. A. Ivanov, A. Putics, T. Hertzig, B. Schelle, S. Bayer, B. Weissbrich, E. J. Snijder, H. Rabenau, H. W. Doerr, A. E. Gorbalenya and J. Ziebuhr: Mechanisms and enzymes involved in SARS coronavirus genome expression. J Gen Virol, 84(Pt 9), 2305-15 (2003) 
10. K. Narayanan, C. Huang and S. Makino: SARS coronavirus accessory proteins. Virus Res, 133(1), 113-21 (2008)

DOI: 10.1016/j.virusres.2007.10.009

PMid:18045721

PMCid:PMC2720074

11. Y. J. Tan, S. G. Lim and W. Hong: Characterization of viral proteins encoded by the SARS-coronavirus genome. Antiviral Res, 65(2), 69-78 (2005)

DOI: 10.1016/j.antiviral.2004.10.001

PMid:15708633

12. Y. J. Tan, S. G. Lim and W. Hong: Understanding the accessory viral proteins unique to the severe acute respiratory syndrome (SARS) coronavirus. Antiviral Res, 72(2), 78-88 (2006)

DOI: 10.1016/j.antiviral.2006.05.010

PMid:16820226

13. K. Sharma, S. Akerstrom, A. K. Sharma, V. T. Chow, S. Teow, B. Abrenica, S. A. Booth, T. F. Booth, A. Mirazimi and S. K. Lal: SARS-CoV $9 \mathrm{~b}$ protein diffuses into nucleus, undergoes active Crm1 mediated nucleocytoplasmic export and triggers apoptosis when retained in the nucleus. PLoS One, 6(5), e19436 (2011)

DOI: 10.1371/journal.pone.0019436

PMid:21637748 PMCid:PMC3103500

14. I. Moshynskyy, S. Viswanathan, N. Vasilenko, V. Lobanov, M. Petric, L. A. Babiuk and A. N. Zakhartchouk: Intracellular localization of the SARS coronavirus protein 9b: evidence of active export from the nucleus. Virus Res, 127(1), 116-21 (2007)

DOI: 10.1016/j.virusres.2007.03.011

PMid:17448558

15. C. S. Shi, H. Y. Qi, C. Boularan, N. N. Huang, M. Abu-Asab, J. H. Shelhamer and J. H. Kehrl: SARS-coronavirus open reading frame-9b suppresses innate immunity by targeting mitochondria and the MAVS/ TRAF3/TRAF6 signalosome. J Immunol, 193(6), 3080-9 (2014)

DOI: 10.4049/jimmunol.1303196

PMid:25135833 PMCid:PMC4179872

16. S. G. Sawicki and D. L. Sawicki: Coronavirus transcription: a perspective. Curr Top Microbiol Immunol, 287, 31-55 (2005)

DOI: 10.1007/3-540-26765-4_2

17. I. Sola, F. Almazan, S. Zuniga and L. Enjuanes: Continuous and Discontinuous
RNA Synthesis in Coronaviruses. Annu Rev Virol, 2(1), 265-88 (2015)

DOI: 10.1146/annurev-virology-100114-

055218

PMid:26958916

18. E. J. Snijder, Y. van der Meer, J. ZevenhovenDobbe, J. J. Onderwater, J. van der Meulen, H. K. Koerten and A. M. Mommaas: Ultrastructure and origin of membrane vesicles associated with the severe acute respiratory syndrome coronavirus replication complex. J Virol, 80(12), 5927-40 (2006)

DOI: 10.1128/JVI.02501-05

PMid:16731931 PMCid:PMC1472606

19. S. Perlman and J. Netland: Coronaviruses post-SARS: update on replication and pathogenesis. Nat Rev Microbiol, 7(6), 43950 (2009)

DOI: $10.1038 / \mathrm{nrmicro} 2147$

PMid:19430490 PMCid:PMC2830095

20. R. W. Chiu, S. S. Chim, Y. K. Tong, K. S. Fung, P. K. Chan, G. P. Zhao and Y. M. Lo: Tracing SARS-coronavirus variant with large genomic deletion. Emerg Infect Dis, 11(1), 168-70 (2005)

DOI: 10.3201/eid1101.040544

PMid:15714661 PMCid:PMC3294368

21. G. M. Pavlovic-Lazetic, N. S. Mitic and M. V. Beljanski: Bioinformatics analysis of SARS coronavirus genome polymorphism. BMC Bioinformatics, 5, 65 (2004)

22. G. M. Pavlovic-Lazetic, N. S. Mitic, A. M. Tomovic, M. D. Pavlovic and M. V. Beljanski: SARS-CoV genome polymorphism: a bioinformatics study. Genomics Proteomics Bioinformatics, 3(1), 18-35 (2005) DOI: 10.1016/S1672-0229(05)03004-4

23. L. L. Poon, C. S. Leung, K. H. Chan, K. Y. Yuen, Y. Guan and J. S. Peiris: Recurrent mutations associated with isolation and passage of SARS coronavirus in cells from nonhuman primates. J Med Virol, 76(4), 435-40 (2005)

DOI: 10.1002/jmv.20379

PMid:15977248

24. J. W. Tang, J. L. Cheung, I. M. Chu, J. J. Sung, M. Peiris and P. K. Chan: The large 386-nt deletion in SARS-associated coronavirus: evidence for quasispecies? J Infect Dis, 194(6), 808-13 (2006) 
25. S. Pfefferle, V. Krahling, V. Ditt, K. Grywna, E. Muhlberger and C. Drosten: Reverse genetic characterization of the natural genomic deletion in SARS-Coronavirus strain Frankfurt-1 open reading frame $7 \mathrm{~b}$ reveals an attenuating function of the $7 \mathrm{~b}$ protein in-vitro and in-vivo. Virol J, 6, 131 (2009)

26. J. A. Robb and C. W. Bond: Pathogenic murine coronaviruses. I. Characterization of biological behavior in vitro and virus-specific intracellular RNA of strongly neurotropic JHMV and weakly neurotropic A59V viruses. Virology, 94(2), 352-70 (1979) DOI: 10.1016/0042-6822(79)90467-7

27. E. Ontiveros, T. S. Kim, T. M. Gallagher and S. Perlman: Enhanced virulence mediated by the murine coronavirus, mouse hepatitis virus strain $\mathrm{JHM}$, is associated with a glycine at residue 310 of the spike glycoprotein. $J$ Virol, 77(19), 10260-9 (2003)

DOI: 10.1128/JVI.77.19.10260-10269.2003

PMid:12970410 PMCid:PMC228498

28. S. Kyuwa, S. Takagaki, S. Matsuyama, F. Taguchi, J. Saegusa, Y. Iwakura, Y. Tagawa and Y. Yoshikawa: Characterization of a variant virus from ascitic fluid of subacute granulomatous serositis in interferongamma-deficient C57BL/6 mice persistently infected with murine coronavirus strain JHM. Viral Immunol, 23(4), 437-42 (2010)

DOI: $10.1089 /$ vim.2010.0008

PMid:20712488

29. F. Y. Zeng, C. W. Chan, M. N. Chan, J. D. Chen, K. Y. Chow, C. C. Hon, K. H. Hui, J. Li, V. Y. Li, C. Y. Wang, P. Y. Wang, Y. Guan, B. Zheng, L. L. Poon, K. H. Chan, K. Y. Yuen, J. S. Peiris and F. C. Leung: The complete genome sequence of severe acute respiratory syndrome coronavirus strain HKU-39849 (HK-39). Exp Biol Med (Maywood), 228(7), 866-73 (2003)

DOI: 10.1177/15353702-0322807-13

30. S. Makino, L. H. Soe, C. K. Shieh and M. M. Lai: Discontinuous transcription generates heterogeneity at the leader fusion sites of coronavirus mRNAs. J Virol, 62(10), 3870-3 (1988)

31. A. O. Pasternak, W. J. Spaan and E. J. Snijder: Nidovirus transcription: how to make sense.? J Gen Virol, 87(Pt 6), 1403$21(2006)$
32. T.J.CowleyandS.R.Weiss:Murinecoronavirus neuropathogenesis: determinants of virulence. J Neurovirol, 16(6), 427-34 (2010) DOI: $10.1007 / B F 03210848$ PMid:21073281 PMCid:PMC3153983

33. T. A. Miura, E. A. Travanty, L. Oko, H. Bielefeldt-Ohmann, S. R. Weiss, N. Beauchemin and K. V. Holmes: The spike glycoprotein of murine coronavirus MHVJHM mediates receptor-independent infection and spread in the central nervous systems of Ceacam1a-/- Mice. J Virol, 82(2), 755-63 (2008)

DOI: $10.1128 / J V I .01851-07$

PMid:18003729 PMCid:PMC2224565

34. C. S. M. E. Consortium: Molecular evolution of the SARS coronavirus during the course of the SARS epidemic in China. Science, 303(5664), 1666-9 (2004)

DOI: 10.1126/science.1092002

PMid:14752165

Abbreviations: Severe Acute Respiratory Syndrome coronavirus (SARS-CoV), subgenomic mRNA (sgmRNA), mouse hepatitis viruses (MHVs), transcriptional regulatory sequences (TRS), core element (CE), double membrane vesicle (DMV), replication and transcription complex (RTC), Dulbecco's modified Eagle's medium (DMEM)

Key Words: SARS-CoV, TRS, sgmRNA, MHV

Send correspondence to: Li Liu, Institute of Basic Medical Sciences, Chinese Academy of Medical Sciences \& School of Basic Medicine, Peking Union Medical College, Beijing 100005, China, Tel: 8610 69156454, Fax: 8610 65233768, E-mail: Iliu@pumc.edu.cn 